\title{
COMMENTS ON OPTICAL STOCHASTIC COOLING
}

\author{
K.Y. NG \\ Fermi National Accelerator Laboratory, P.O. Box 500, Batavia, IL 60510, USA \\ E-mail: ng@fnal.gov \\ S.Y. LEE AND Y.K. ZHANG \\ Department of Physics, Indiana University, Bloomington, IN 47405, USA \\ E-mail: shylee@indiana.edu,yzhang2@indiana.edu
}

\begin{abstract}
An important necessary condition for transverse phase space damping in the optical stochastic cooling with transit-time method is derived. The longitudinal and transverse damping dynamics for the optical stochastic cooling is studied. We also obtain an optimal laser focusing condition for laser-beam interaction in the correction undulator. The amplification factor and the output peak power of the laser amplifier are found to differ substantially from earlier publications. The required power is large for hadron colliders at very high energy.
\end{abstract}

\section{Introduction}

Transit-time optical stochastic cooling (OSC) was first introduced by Zolotorev and Zholents ${ }^{1}$, where the optical frequency of $\sim 3 \times 10^{5} \mathrm{GHz}(\lambda \sim 1 \mu \mathrm{m})$ is used. This provides a bandwidth more than ten thousand times larger than microwave stochastic cooling ${ }^{2}$. The OSC can be used in low energy electron rings to provide high brightness beams. It can also be used in proton collider rings to increase luminosity by counteracting intra-beam scattering.

In Ref. 1, the horizontal cooling decrement is presented as

$\alpha_{x}=\frac{1}{2}\left(\frac{\overline{\Delta\left(x^{2}\right)}}{\overline{x^{2}}}+\frac{\overline{\Delta\left(x^{\prime 2}\right)}}{\overline{x^{\prime 2}}}\right)=\frac{1}{2}\left[4 G D_{0} \eta_{0}^{\prime} k \exp \left\{-\frac{\overline{\Delta \phi_{i}}}{2}\right\}-\frac{G^{2} N_{s}}{2}\left(\eta_{0}^{\prime 2}+\frac{D_{0}^{2}}{\beta^{2}}\right) \frac{\beta}{\epsilon_{x}}\right]$,

where, in their notations, $D_{0}$ and $-\eta_{0}^{\prime}$ represent the dispersion and dispersion gradient at the second undulator. In above, the first term on the right represents cooling while the second term represents heating. Thus, there is no horizontal cooling if $\eta_{0}^{\prime}=0$, which can hardly be correct. In order to understand OSC, we rederived all the equations of the dynamic again. The detail is given in Ref 3 .

\section{Transit-time OSC}

In the transit-time OSC, the beam particles pass through a first undulator where photons are emitted. After amplification by a laser amplifier, these photons meet the beam particles again inside the second undulator and interact with them by correcting their momentum offset. In order to receive the right corrections, the phases of the beam particles are properly adjusted by allowing the beam particles traveling through a bypass as shown in Fig. 1.

Consider the $i$-th beam particle with a momentum deviation $\delta_{i}=\Delta P_{i} / P$, and the betatron phase space coordinates $\left(x_{i 1}, x_{i 1}^{\prime}, z_{i 1}, z_{i 1}^{\prime}\right)$ at the first undulator. In the 


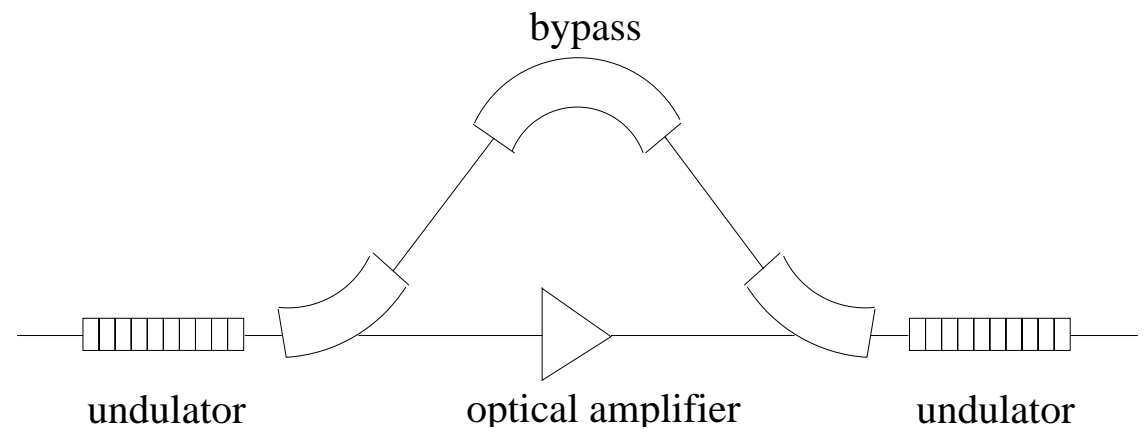

Figure 1. The module for transit time OSC, which consists of two undulators, a laser amplification system, and a by-pass for the beam particles.

Frenet-Serret coordinate system, the path length of the test particle in the bypass section is ${ }^{4}$

$$
\ell_{i}=\int_{s_{1}}^{s_{2}} \sqrt{\tilde{x}_{i}^{\prime 2}+\tilde{z}_{i}^{\prime 2}+\left(1+\frac{\tilde{x}_{i}}{\rho}\right)^{2}} d s \approx \int_{s_{1}}^{s_{2}}\left(1+\frac{\tilde{x}_{i}}{\rho}\right) d s,
$$

where $\hat{x}, \hat{s}$ and $\hat{z}$ form a curvilinear coordinate system with a horizontal bending radius $\rho$, the coordinates $\tilde{x}_{i}$ and $\tilde{z}_{i}$ are the deviations from a reference orbit, and $s$ is the longitudinal coordinate along the reference orbit. We have also assumed $\tilde{x}_{i}^{\prime} \ll 1$ and $\tilde{z}_{i}^{\prime} \ll 1$ to obtain the last approximate equality. For a bypass with planar geometry, the transverse displacement of an orbiting particle is given by $\tilde{x}_{i}(s)=$ $x_{\text {co }}(s)+M_{11}\left(s, s_{1}\right) x_{i 1}+M_{12}\left(s, s_{1}\right) x_{i 1}^{\prime}+D(s) \delta_{i}$, where $M_{11}\left(s, s_{1}\right)$ and $M_{12}\left(s, s_{1}\right)$ are transport matrix elements of the Hill's equation from the first undulator at $s_{1}$ to the second undulator at $s_{2}$ via the beam bypass, $x_{\mathrm{co}}(s)$ is the closed orbit around the reference orbit, and $D(s)$ is the dispersion function. The path length for the $i$-th particle in the bypass region becomes

$$
\ell_{i}=\ell_{0}+x_{i 1} I_{1}+x_{i 1}^{\prime} I_{2}+\delta_{i} I_{D}
$$

where $x_{i 1}, x_{i 1}^{\prime}$ are the conjugate phase space coordinates for the $i$-th particle at the location $s_{1}$, and the integrals $I_{1}, I_{2}$, and $I_{D}$ are

$$
I_{1}=\int_{s_{1}}^{s_{2}} \frac{M_{11}\left(s, s_{1}\right) d s}{\rho(s)}, \quad I_{2}=\int_{s_{1}}^{s_{2}} \frac{M_{12}\left(s, s_{1}\right) d s}{\rho(s)}, \quad I_{D}=\int_{s_{1}}^{s_{2}} \frac{D(s) d s}{\rho(s)} .
$$

The on-momentum particle arrives at the second undulator and sees exactly the zero crossing of the electric field of the photons. The $i$-th particle, however, arrives with a time delay and therefore at a phase

$$
\Delta \phi_{i}=k\left(\ell_{i}-\ell_{0}\right)=k\left(x_{i 1} I_{1}+x_{i 1}^{\prime} I_{2}+\delta_{i} I_{D}\right)
$$

from the zero-crossing of the electric field, where $k$ is the wavenumber of the photons. The correction through the electric field is ${ }^{5}$

$$
\Delta \delta_{i}=-\operatorname{sgn}\left(I_{D}\right) G \sin \left(\Delta \phi_{i}\right),
$$

osc-talk: submitted to World Scientific on October 23, 2002 
where the gain factor is

$$
G=\frac{q\left\langle\mathcal{E}_{2}\right\rangle N_{u} \lambda_{u} K[J J]}{2 \gamma E_{b}}
$$

$K=q B_{u} \lambda_{u} /(2 \pi m c)$ is the strength parameter of the undulator with field strength $B_{u}$, wavelength $\lambda_{u}$, and number of period $N_{u}, q$ is the charge of the beam particle with nominal energy $E_{b}=\gamma m c^{2}$ and rest mass $m, \mathcal{E}_{2}$ is the electric field amplitude of the electromagnetic wave and \langle\rangle represents its average along the second undulator, $c$ is the velocity of light, $[J J]=J_{0}\left(\frac{1}{2} \xi\right)-J_{1}\left(\frac{1}{2} \xi\right)$ with $\xi=K^{2} /\left(2+K^{2}\right)$, and $J_{0}$ and $J_{1}$ are Bessel functions. Through the dispersion $D_{2}$ at second undulator, there are horizontal corrections $\Delta x_{i}=-D_{2} \Delta \delta_{i}, \Delta x_{i}^{\prime}=-D_{2}^{\prime} \Delta \delta_{i}$. Thus there is also horizontal cooling.

\section{Damping Decrements}

The correction in Eq. (6) is the result of the interaction of the photons emitted by a beam particle on the same beam particle. This interaction is coherent and produces a damping. However, this particle also interacts with the photons emitted by other beam particles. This interaction is incoherent and produces a growth instead. Adding up cooling and anti-cooling, the resulting longitudinal damping decrement is

$$
\alpha_{\delta} \equiv-\frac{\left\langle\delta_{i c}^{2}-\delta_{i}^{2}\right\rangle}{\sigma_{\delta}^{2}}=2 G k I_{D} e^{-u}-\frac{G^{2} N_{s}}{2 \sigma_{\delta}^{2}}
$$

where

$$
u=\frac{1}{2} k^{2}\left[\left(\beta_{1} I_{1}^{2}-2 \alpha_{1} I_{1} I_{2}+\gamma_{1} I_{2}^{2}\right) \epsilon_{x}+I_{D}^{2} \sigma_{\delta}^{2}\right]
$$

is the total thermal energy of the system, with $\beta_{1}, \alpha_{1}$, and $\gamma_{1}$ the corresponding Twiss parameters at the first undulator. Here, a Gaussian momentum distribution has been assumed for the beam particles at the first undulator with $\sigma_{\delta}$ being the rms momentum spread.

For the horizontal, we introduce the normalized coordinates $\left(x, P_{x}=\beta x^{\prime}+\alpha x\right)$. The horizontal damping decrement is just

$$
\alpha_{x} \equiv-\frac{\left\langle P_{x 2 c}^{2}+x_{2 c}^{2}-\left(P_{x 2}^{2}+x_{2}^{2}\right)\right\rangle}{\sigma_{x 2}^{2}}=2 G k I_{\perp} e^{-u}-\frac{G^{2} N_{s} \mathcal{H}_{2}}{2 \epsilon_{x}},
$$

where

$$
\mathcal{H}_{2}=\frac{D_{2}^{2}+P_{D 2}^{2}}{\beta_{2}}
$$

is the $\mathcal{H}$-function at 2 nd undulator, and

$$
\begin{aligned}
I_{\perp}= & -\frac{\beta_{1}}{\beta_{2}}\left\{P _ { D 2 } \left[\left(\left(\beta_{2} M_{21}+\alpha_{2} M_{11}\right)-\frac{\alpha_{1}}{\beta_{1}}\left(\beta_{2} M_{22}+\alpha_{2} M_{12}\right)\right)\left(I_{1}-\frac{\alpha_{1}}{\beta_{1}} I_{2}\right)\right.\right. \\
& \left.\left.+\frac{\beta_{2} M_{22}+\alpha_{2} M_{12}}{\beta_{1}^{2}} I_{2}\right]+D_{2}\left[\left(M_{11}-\frac{\alpha_{1}}{\beta_{1}} M_{12}\right)\left(I_{1}-\frac{\alpha_{1}}{\beta_{1}} I_{2}\right)+\frac{M_{12}}{\beta_{1}^{2}} I_{2}\right]\right\} .
\end{aligned}
$$

osc-talk: submitted to World Scientific on October 23, 2002 
We have introduced for the $i$-th particle at the second undulator the normalized betatron coordinate $P_{x i 2}=\beta_{2} x_{i 2}^{\prime}+\alpha_{2} x_{i 2}$ and the normalized dispersion phase space coordinate $P_{D 2}=\beta_{2} D_{2}^{\prime}+\alpha_{2} D_{2}$. Thus, we arrive at the necessary condition of horizontal damping: $I_{\perp}>0$. Notice that transverse cooling can still be possible even when $D_{2}=0$.

\section{Cooling Dynamic}

From the damping decrements, it is easy to compute the rates at which the transverse emittance and the momentum are damped:

$$
\begin{aligned}
\frac{d \epsilon_{x}}{d t} & =-\frac{2 G k I_{\perp} \epsilon_{x}}{T_{0}} e^{-u}+\frac{G^{2} N_{s} \mathcal{H}_{2}}{2 T_{0}}, \\
\frac{d \sigma_{\delta}^{2}}{d t} & =-\frac{2 G k I_{D} \sigma_{\delta}^{2}}{T_{0}} e^{-u}+\frac{G^{2} N_{s}}{2 T_{0}} .
\end{aligned}
$$

In the special case of $I_{D}=I_{\perp}$, there is equal gain in both the transverse and longitudinal directions. We can then combine Eqs. (13) and (14) to obtain

$$
\frac{d u}{d t}=-\frac{2 G_{0} k I_{D}}{T_{0}} u e^{-u}+\frac{G_{0}^{2} N_{s} v}{2 T_{0}},
$$

where

$$
v=\frac{1}{2} k^{2}\left[\left(\beta_{1} I_{1}^{2}-2 \alpha_{1} I_{1} I_{2}+\gamma_{1} I_{2}^{2}\right) \mathcal{H}_{2}+I_{D}^{2}\right] .
$$

The optimum gain is

$$
\frac{d u}{d t}=-\frac{2 k^{2} I_{D}^{2}}{v N_{s} T_{0}} u^{2} e^{-2 u},
$$

which occurs when

$$
G_{\mathrm{opt}}=\frac{2 k I_{D}}{v N_{s}} u e^{-u} .
$$

The cooling of the thermal energy $u$ at optimized gain is shown in Fig. 2 starting from the initial value of $u_{0}=3$. Because of the $u^{2} e^{-2 u}$ factor, the cooling slows down as time progresses. When $u \leq 1$, the cooling process behaves like $u=\frac{1}{t}$ and becomes very inefficient. However, the OSC takes place through Eq. (6), which is proportional to $\sin \left(\Delta \phi_{i}\right)$, and the correction will be in the wrong direction if the phase shift $\left|\Delta \phi_{i}\right|>\pi / 2$. Thus, for a large thermal energy, like $u_{0}=3$, only the part of the beam sufficiently close to the on-momentum particle will be cooled while the rest will be heated instead. To ensure OSC, we must make sure that all the particles in the beam (usually $95 \%$ is assumed) be within the $\pi / 2$ phase shift. Since a bypass can be designed with very small $I_{1}$ and $I_{2}$, this phase shift requirement translates into

$$
u=u_{0} \approx \frac{1}{2}\left(k I_{D} \sigma_{\delta}\right)^{2} \leq \frac{\pi^{2}}{48} .
$$

As a result, OSC at optimum gain factor is rather inefficient because the emittance of a cold beam decreases inversely with the cooling time. As will be seen below,

osc-talk: submitted to World Scientific on October 23, 2002 


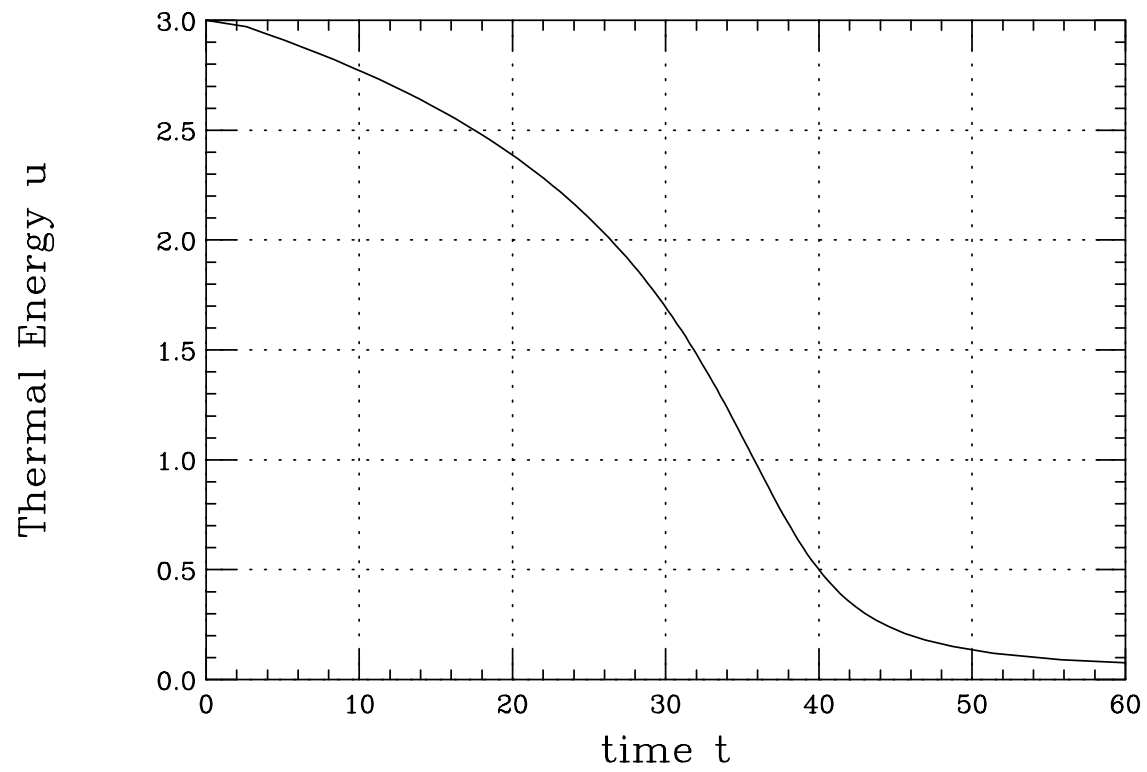

Figure 2. The cooling of the thermal energy $u$ from the initial value $u_{0}=3$, obtained by solving the Eq. (17) is shown as a function of time $t$ with optimal gain factor.

OSC at small gain turns out to be more efficient. Although the cooling represented by Eq. (17) is not exponential, an initial cooling time can nevertheless be defined by

$$
\tau_{\text {cool }}=-\left.\frac{u}{d u / d t}\right|_{u=u_{0}} \approx \frac{N_{s} T_{0}}{4} \frac{e^{2 u_{0}}}{u_{0}}
$$

for an optimum gain factor.

\section{Peak Power}

After being amplified by the laser amplifier and focused to the middle of the second undulator, the electromagnetic pulse of the emitted photons at the waist has a time duration of $\Delta t_{R}=N_{u} \lambda / c$, an electric field amplitude $\mathcal{E}_{2}$ and an area of cross section $A_{2}$. The total energy of the electromagnetic pulse is

$$
W_{2}=\frac{1}{2} \epsilon_{0} \mathcal{E}_{2}^{2} A_{2} c \Delta t_{R}
$$

where $\epsilon_{0}$ is the electric permittivity of free space. The output peak power of the laser amplifier is therefore

$$
\hat{P}_{2}=\frac{W_{2}}{\Delta t_{R}} N_{s}=\frac{1}{2} \epsilon_{0} \mathcal{E}_{2}^{2} A_{2} c N_{s},
$$


where

$$
N_{s}=N_{B} \frac{N_{u} \lambda}{2 \sqrt{6} c \sigma_{\tau}}
$$

is the number of particles in a sample within a bandwidth of $\left.\Delta \omega\right|_{\mathrm{FWHM}}=\omega / N_{u}$, and $\sigma_{\tau}$ is the rms length of a bunch containing $N_{B}$ beam particles to be cooled. Here, we have assumed $100 \%$ photon transmission in the optical amplifier, and assume that the bandwidth of the laser amplifier is larger than that of the undulator radiation.

To relate the peak output power to the gain factor $G$ defined in Eq. (7), we need to compute the electric field amplitude averaged along the second undulator. Since the energy of the electromagnetic pulse must be the same as it travels down the second undulator, we must have

$$
\mathcal{E}_{2}(s) A_{2}(s)=\mathcal{E}_{2} A_{2} .
$$

On the other hand, diffraction requires the cross-sectional area

$$
A_{2}(s)=A_{2}\left(1+\frac{s^{2}}{Z_{R}^{2}}\right),
$$

where $Z_{R}=\sigma_{r} / \sigma_{r^{\prime}}$, the ratio of the transverse size of the electromagnetic wave to its divergence, is called the Rayleigh length, which just plays the role of $\beta^{*}$, the minimum betatron function in the situation of a particle beam. We therefore have

$$
\left\langle\mathcal{E}_{2}\right\rangle=\frac{2 \mathcal{E}_{2}}{N_{u} \lambda_{u}} \int_{0}^{N_{u} \lambda_{u} / 2} \frac{d s}{\sqrt{1+\left(s / Z_{R}\right)^{2}}},
$$

where $N_{u} \lambda_{u}$ is the length of the second undulator. Combining Eqs. (7), (22), and (26), we arrive at the peak power for a given gain factor,

$$
\hat{P}_{2}=G^{2} \frac{N_{s}\left(E_{b} / q\right)^{2}}{Z_{0} \xi N_{u}[J J]^{2}} \mathcal{F}_{2}
$$

where $Z_{0}$ is the impedance of free space,

$$
\mathcal{F}_{2}=\frac{A_{0} / A_{2}}{8\left[\ln \left(A_{0} / A_{2}+\sqrt{1+\left(A_{0} / A_{2}\right)^{2}}\right)\right]^{2}},
$$

$A_{2}=2 \pi \sigma_{r}^{2}$ is the photon beam area at the waist of the second undulator ${ }^{6}$ as defined in Eq. (21), and $A_{0}=N_{u} \lambda_{u} \lambda / 4$. Minimum laser amplifier power occurs ${ }^{7}$ when $A_{2}=0.3012 A_{0}$, where $\mathcal{F}_{2}=0.1132$.

The average laser power is equal to the peak power multiplied by the duty factor, i.e.,

$$
\langle P\rangle_{2}=\hat{P}_{2} \frac{n_{b} 2 \sqrt{6} \sigma_{\tau}}{T_{0}}=G^{2} \frac{\left(E_{b} / q\right)^{2}}{Z_{0} \xi[J J]^{2}} \frac{N_{B} n_{b} \lambda}{C} \mathcal{F}_{2},
$$

where $n_{b}$ is the number of bunches, $\sigma_{\tau}$ is the rms bunch length in time, and $C$ is the circumference of the storage ring. Note that the average power is proportional to the total number of particles in the storage ring.

osc-talk: submitted to World Scientific on October 23, 2002 


\subsection{Peak Laser power for optimal gain}

Substituting the optimal gain of Eq. (18) into Eq. (28), we obtain the output peak power of the laser amplifier given by

$$
\hat{P}_{2}=\frac{N_{s}\left(E_{b} / q\right)^{2}}{Z_{0} \xi[J J]^{2} N_{u}}\left(\frac{2 k I_{D}}{v N_{s}} u e^{-u}\right)^{2} \mathcal{F}_{2}
$$

Since the cooling rate is inversely proportional to $N_{s}$, the peak power for an optimized cooling of $N_{s}$ particle is also inversely proportional to $N_{s}$. Because of the stability condition of $u \leq \pi^{2} / 48$ in Eq. (19), the peak power is highly reduced.

\section{Application to Hadron Machines}

Figure 3 shows the peak power versus $\gamma$ (beam energy) for proton storage rings at optimal gain. The laser wavelength is taken to be $\lambda=1 \mu \mathrm{m}$ and each undulator has

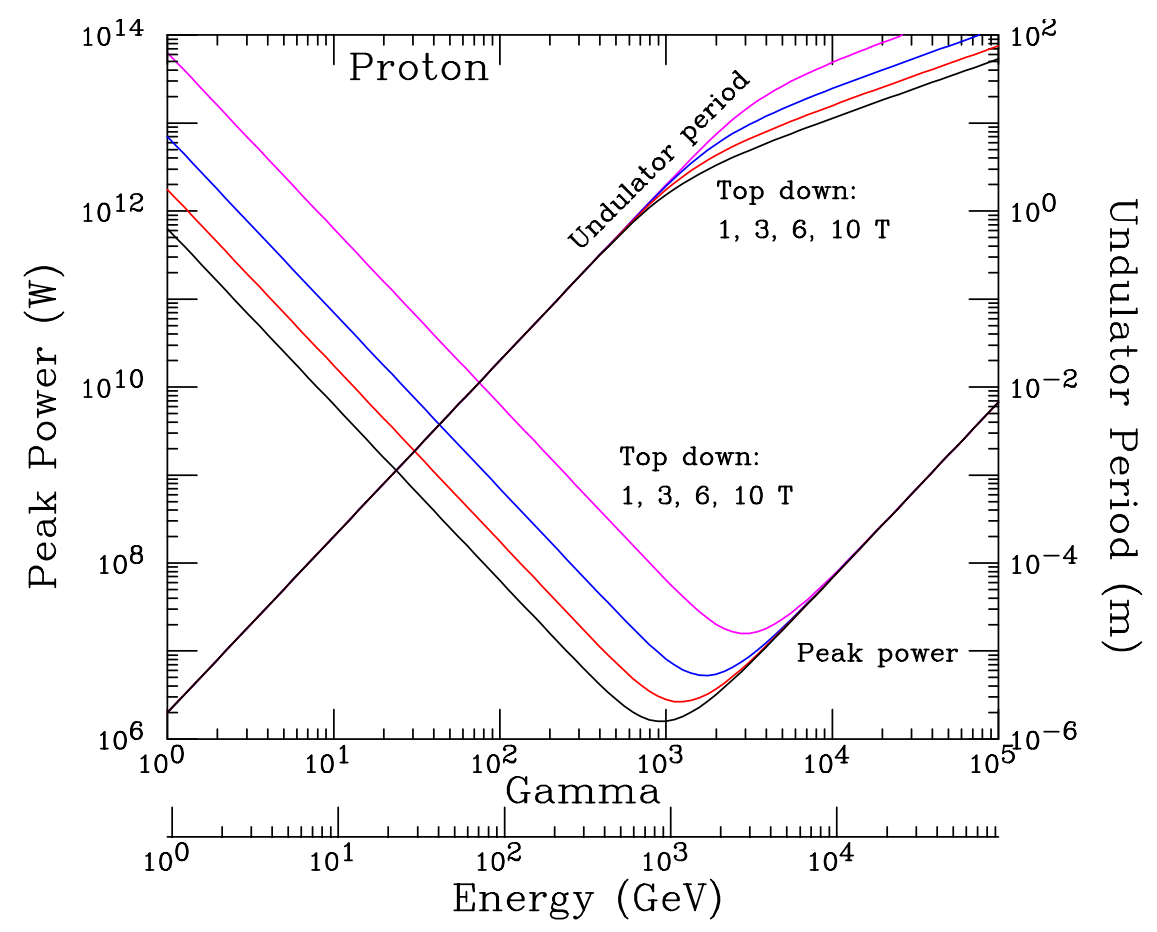

Figure 3. The peak laser amplifier power vs $\gamma$ for an optimal gain in the optical stochastic cooling for a proton storage ring (TEVATRON). The parameters for the TEVATRON are $\sigma_{\ell}=0.37 \mathrm{~m}$, $\sigma_{\delta}=1.3 \times 10^{-4}, n_{b}=36$ bunches, each containing $N_{B}=2.7 \times 10^{11}$ particles, $E_{b}=1 \mathrm{TeV}$, the mean radius of the TEVATRON of $1000 \mathrm{~m}$, and $B_{u}=10 \mathrm{~T}$. The initial cooling time is given by Eq (20) with $u_{0}=\pi^{2} / 48$ or $\tau_{\text {cool }} \approx 57 \mathrm{~s}$.

$N_{u}=10$ periods. Most parameters correspond to the TEVATRON at Fermilab: $N_{B}=2.7 \times 10^{11}$ particles, $r m s$ bunch length $\sigma_{\ell}=0.37 \mathrm{~m}$, and $\sigma_{\delta}=1.3 \times 10^{-4}$.

osc-talk: submitted to World Scientific on October 23, 2002 
With the TEVATRON revolution period of $T_{0}=20.96 \mu \mathrm{s}$, the initial cooling time is $57 \mathrm{~s}$ given by Eq. (20). The magnetic field of the undulator varies from 1 to $10 \mathrm{~T}$.

For a fixed laser wavelength and the undulator magnetic field, the undulator parameter is obtained by solving the cubic equation:

$$
\lambda=\frac{\pi m c}{2 q B_{u} \gamma^{2}} K\left(2+K^{2}\right),
$$

from which the undulator period $\lambda_{u}$ can be solved and plotted in Fig. 3. The selfconsistent solution gives $K \sim \gamma^{2}$ at low energies and $\hat{P}_{2} \sim \frac{\left(E_{b} / q\right)^{2}}{\xi} \sim \frac{\left(E_{b} / q\right)^{2}}{K^{2}} \sim \frac{1}{\gamma^{2}}$; i.e., it requires a large laser power to compensate the small coherent radiation flux for hadron beams at low energies. At high energies, the particle beam is stiff and the number of photons emitted in the solid angle $\lambda /\left(N_{u} \lambda_{u}\right)$ becomes saturated $(\xi \rightarrow 1)$. The output power increases as $\gamma^{2}$ instead. The position of the minimum laser power can be easily calculated to be

$$
\gamma_{\min }=\sqrt{\frac{4 \sqrt{2} \pi}{3 \sqrt{3}}} \sqrt{\frac{m c}{q B_{u} \lambda}} .
$$

The TEVATRON at $1 \mathrm{TeV}$ happens to be near the minimum of the power-vs-gamma curve and is therefore favored ${ }^{8}$ by OSC. The undulator period of $\lambda_{u}=1.93 \mathrm{~m}$ $\left(B_{u}=6 \mathrm{~T}\right)$ is long enough for superconducting undulators. The Relativistic Hadron Collider (RHIC) lies on the left side of the minimum and has its output amplifier power scale as $\gamma^{-2}(m / q)^{4}$. The Very Large Hadron Collider (VLHC) lies on the right side of the minimum and has its output power scale as $(m \gamma / q)^{2}$.

\section{Application to Electron Machines}

Figure 4 is a similar plot for electron rings. Because of the small electron mass, there is no need to consider high magnetic field undulators and we set the magnetic field at $B_{u}=1 \mathrm{~T}$. The bunch parameters are $N_{B}=1.0 \times 10^{11}, \sigma_{\ell}=1 \mathrm{~cm}$, and $\sigma_{\delta}=1.3 \times 10^{-4}$. Besides laser wavelength $\lambda=1 \mu \mathrm{m}$, we also include $\lambda=5,20$, and $100 \mu \mathrm{m}$, where the corresponding numbers of sampling particle are $N_{s}=2.0 \times 10^{7}$, $1.0 \times 10^{8}, 4.1 \times 10^{8}$, and $2.0 \times 10^{9}$ respectively. The initial cooling time for the optimal gain given by Eq. (20) is $\tau_{\text {cool }}=1.8 N_{s} T_{0}$, which depends on the revolution period $T_{0}$.

When $\lambda=1 \mu \mathrm{m}$, the minimum peak power occurs at $\gamma_{\min }=76.3$ or $E_{b}=$ $39.0 \mathrm{MeV}$; i.e., nearly all electron storage rings lie on the right side of the minimum. However, because of the $(\mathrm{m} / \mathrm{q})^{2}$ factor, the output power of the amplifier is very much reduced. That does not imply that OSC favors electron rings of high energies because the radiation damping rate increases rapidly with energy. To be effective, the OSC cooling rate, discussed in the last paragraph, has to be faster than the radiation damping rate of the electron ring.

Now, we consider a possible example of converting the Cooler Ring at the Indiana University Cyclotron Facility (IUCF) to an electron ring and OSC is applied at the Ti-Saphire laser wavelength $\lambda=0.78 \mu \mathrm{m}$ with $N_{u}=10$ and $\lambda_{u}=5 \mathrm{~cm}$. Setting an initial cooling time of $0.10 \mathrm{~s}$, we find $N_{s}=1.92 \times 10^{5}$. Since the rms bunch length is $3.6 \mathrm{~cm}$ with the $\mathrm{rf}$ system, we find the number of particles in a bunch

osc-talk: submitted to World Scientific on October 23, 2002 


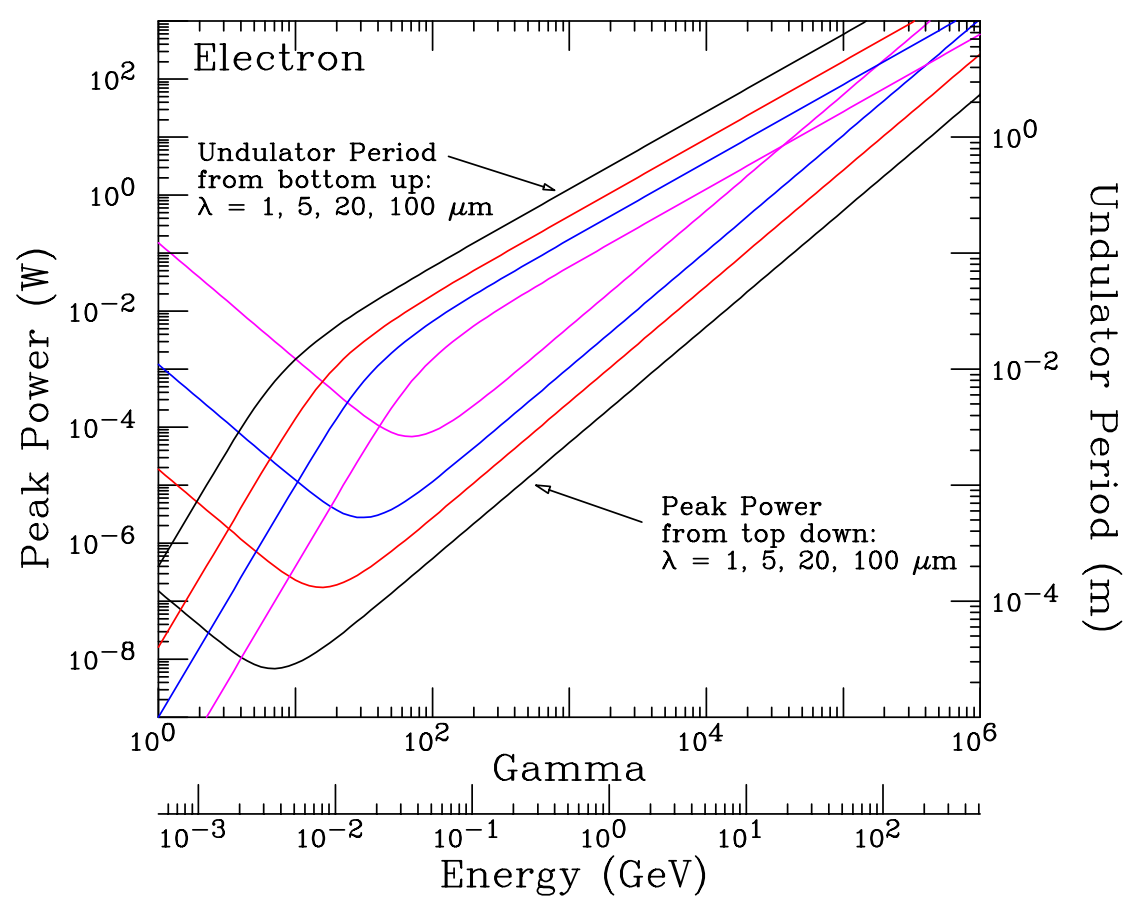

Figure 4. The peak laser amplifier power vs $\gamma$ for optimal gain in the optical stochastic cooling for electron storage rings. The parameters for the electron storage ring are $\sigma_{\ell}=1 \mathrm{~cm}, \sigma_{\delta}=1.3 \times 10^{-4}$, $N_{B}=1.0 \times 10^{11}$, and $B_{u}=1.0 \mathrm{~T}$.

$N_{B}=4.36 \times 10^{9}$. At $E_{b}=500 \mathrm{MeV}$, the required laser peak power is $\hat{P}=39 \mathrm{~W}$. The peak power is much larger than that of Fig. 4 because the number of the sampling particle is much smaller in this example. The natural horizontal emittance and the OSC-equilibrium emittance are plotted in Fig. 5 as functions of beam energy. Other parameters used in the plots are ring circumference $C=85.03 \mathrm{~m}$, bending radius $\rho=2.44 \mathrm{~m}$, momentum compaction $\alpha_{c}=0.04938$, rf harmonic $h=15$, and a bucket-to-bunch-height ratio of 40 . We note that the emittance is OSC-damped by almost an order of magnitude or more when the electron energy is below $500 \mathrm{MeV}$. However, at higher energies, OSC damping is completely inefficient because of the rapidly increasing radiation damping rates. As a whole, applications of OSC to low energy electron storage rings can be useful for attaining high brightness electron beams.

\section{Laser Power for Low-Gain Regime}

At an optimal gain, the laser power requirement is usually high (see Fig. 3), and the damping dynamics is not necessarily the most favorable for beam cooling. It would be useful to consider the OSC in the low-gain regime. As an example, if the second undulator location is designed to be non-dispersive, i.e. $D_{2}=P_{D 2}=0$,

osc-talk: submitted to World Scientific on October 23, 2002 


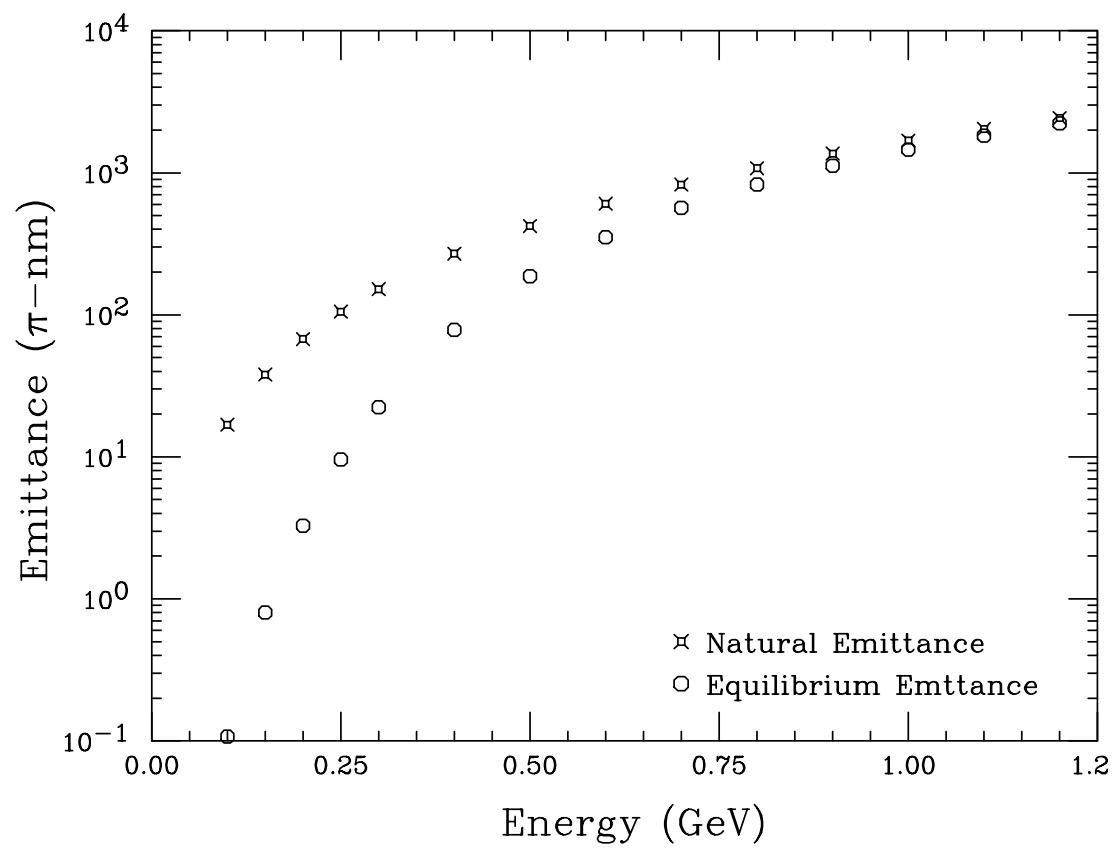

Figure 5. The equilibrium electron emittance for a cooling time of $0.1 \mathrm{~s}$ is shown as a function of the electron beam energy.

the betatron cooling and heating vanish. The OSC becomes a one-dimensional momentum cooling device and the cooling bypass design is simplified. Let $u_{x}=$ $\frac{1}{2} k^{2}\left(\beta_{1} I_{1}^{2}-2 \alpha_{1} I_{1} I_{2}+\gamma_{1} I_{2}^{2}\right) \epsilon_{x}$, and $u_{\delta}=\frac{1}{2} k^{2} I_{D}^{2} \sigma_{\delta}^{2}$. In the low-gain regime, the incoherent heating term is now small and can be neglected. The damping equation becomes

$$
\frac{d u_{\delta}}{d t}=-\frac{2 G k I_{D}}{T_{0}} e^{-u_{x}} u_{\delta} e^{-u_{\delta}} .
$$

Since $u_{\delta} \leq \pi^{2} / 48$ is small, the damping is almost exponential and becomes more so as the cooling proceeds and will continue until the cooling force is balanced by the heating forces coming from rf noise, intra-beam scattering, etc. This is highly in contrast with the cooling at optimum gain-factor discussed in Sec. III.C.2, where the cooling process becomes more and more inefficient as the beam is cooled. With $u_{x}=0$, the cooling time is

$$
\tau_{\text {cool }} \approx \frac{e_{\delta}^{u}}{2 G k I_{D}} T_{0} .
$$

The resulting peak power is

$$
\hat{P}_{2}=\left(\frac{T_{0}}{\tau_{\text {cool }}}\right)^{2} \frac{N_{s}\left(E_{b} / q\right)^{2} e^{2 u_{\delta}}}{Z_{0} N_{u} \xi[\mathrm{JJ}]^{2}\left(2 k I_{D}\right)^{2}} \mathcal{F}_{2},
$$




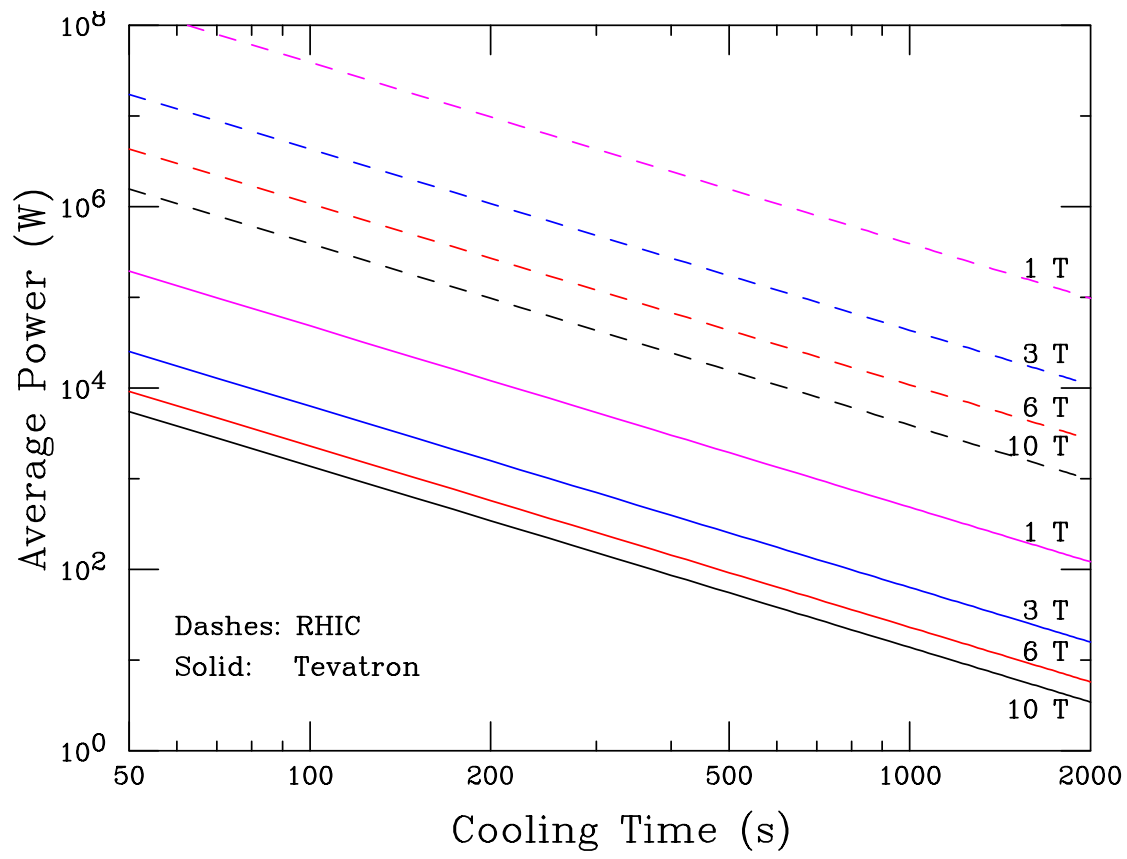

Figure 6. The laser amplifier power in the low gain regime for the TEVATRON at $1 \mathrm{TeV}$ and the RHIC at $100 \mathrm{GeV} / \mathrm{amu}$. The laser wavelength is $\lambda=1 \mu$, and the undulator parameters are $N_{u}=$ 10 with the magnetic field strength $B_{u}$ listed in the graph. The corresponding beam parameters are $\sigma_{\ell}=0.37 \mathrm{~m}, \sigma_{\delta}=1.3 \times 10^{-4}$, and $n_{b}=36$ bunches, each containing $N_{B}=2.7 \times 10^{11}$ particles, at $E_{b}=1 \mathrm{TeV}$ for the TEVATRON; and $\sigma_{\tau}=2 \mathrm{~ns}, \sigma_{\delta}=1.0 \times 10^{-3}, n_{b}=60$ bunches, each containing $N_{B}=1.0 \times 10^{9}$ particles, $E_{b}=100 \mathrm{GeV} /$ nucleon for gold ion, and the circumference of $3833.85 \mathrm{~m}$ for the RHIC.

The average power of the laser amplifier is

$$
\langle P\rangle_{2}=\left(\frac{T_{0}}{\tau_{\text {cool }}}\right)^{2}\left(\frac{n_{b} N_{B} \lambda}{C}\right) \frac{\left(E_{b} / q\right)^{2} e^{2 u_{\delta}}}{Z_{0} \xi[\mathrm{JJ}]^{2}\left(2 k I_{D}\right)^{2}} \mathcal{F}_{2} .
$$

Note that the average power depends on the total number of particles $n_{b} N_{B}$ in the ring and the square of the energy over charge $\left(E_{b} / q\right)^{2}$.

Figure 6 shows the average power requirement versus cooling time in the low gain regime, where the undulator parameters are $\lambda=1.0 \mu \mathrm{m}, N_{u}=10$, and the undulator magnetic field varying from $1 \mathrm{~T}$ to $10 \mathrm{~T}$. The corresponding beam parameters are $\sigma_{\ell}=0.37 \mathrm{~m}, \sigma_{\delta}=1.3 \times 10^{-4}, n_{b}=36$ bunches each containing $N_{B}=2.7 \times 10^{11}$ protons at $E_{b}=1 \mathrm{TeV}$ for the TEVATRON whose mean radius is $1 \mathrm{~km}$, while $\sigma_{\tau}=2.0 \mathrm{~ns}, \sigma_{\delta}=1.0 \times 10^{-3}, n_{b}=60$ bunches each containing $N_{B}=1.0 \times 10^{9}$ gold ions $(A=197$ and $Z=79)$ at $E_{b}=100 \mathrm{GeV} /$ nucleon for the RHIC whose circumference is $3833.85 \mathrm{~m}$. We see that for a cooling time of $1200 \mathrm{~s}$ which is fast enough to counteract intra beam scattering, the average output power for the TEVATRON is only $16 \mathrm{~W}$ when superconducting undulators at $B_{u}=6 \mathrm{~T}$ are used. On the other hand, the average output power for the RHIC is more than

osc-talk: submitted to World Scientific on October 23, 2002 


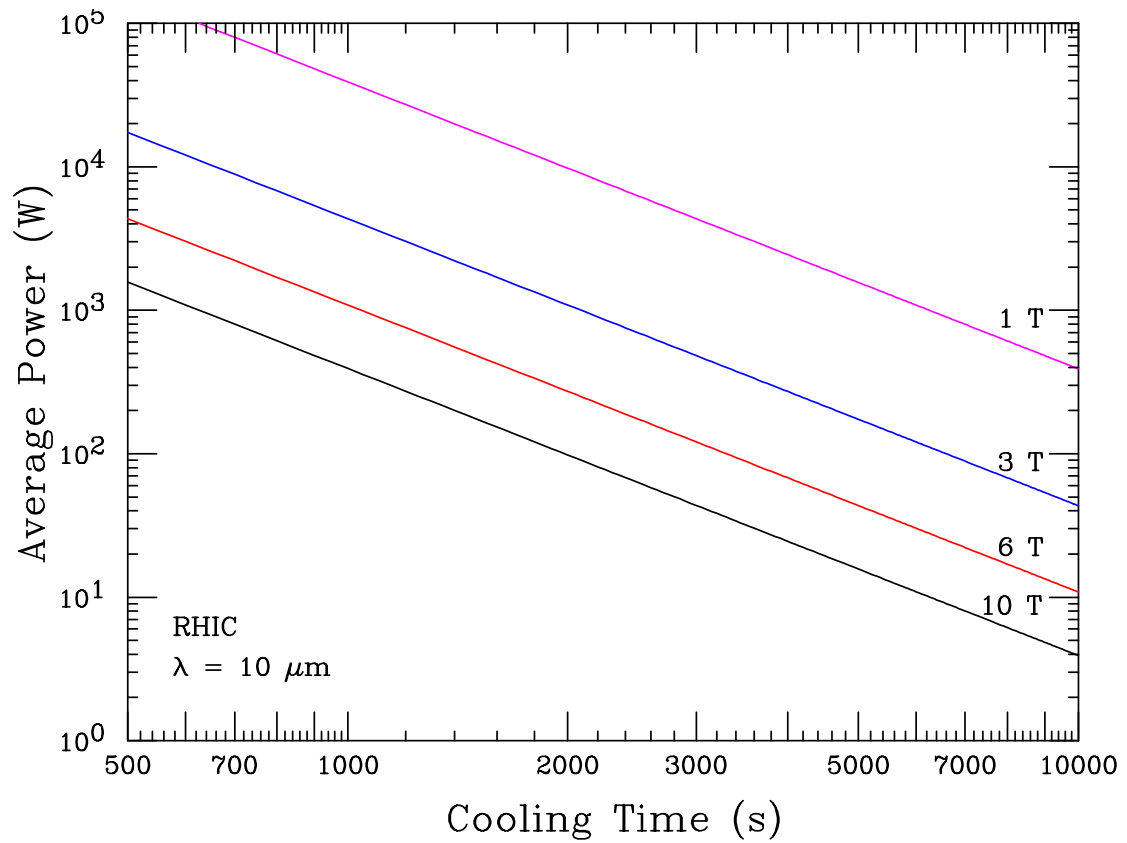

Figure 7. The laser amplifier power in the low gain regime for the RHIC at $100 \mathrm{GeV} / \mathrm{amu}$ with the laser wavelength of $\lambda=10 \mu$ and the undulator parameters are $N_{u}=10$. The magnetic field strength $B_{u}$ is listed in the graph. The corresponding beam parameters are $\sigma_{\tau}=2 \mathrm{~ns}, \sigma_{\delta}=$ $1.0 \times 10^{-3}, n_{b}=60$ bunches, each containing $N_{B}=1.0 \times 10^{9}$ particles, $E_{b}=100 \mathrm{GeV} / \mathrm{nucleon}$ for gold ion, and the circumference of $3833.85 \mathrm{~m}$.

1000 times larger. Because $\gamma$ is one order of magnitude smaller than that of the TEVATRON, the undulator period becomes $\lambda_{u}=2.3 \mathrm{~cm}$, two orders of magnitude smaller. This implies that superconducting undulators may not be used and only $1 \mathrm{~T}$ undulators are possible. The output power for the RHIC application is therefore increased at least one more order of magnitude.

Note that when the laser wavelength is chosen to be $\lambda=1 \mu \mathrm{m}$ for the RHIC, the undulator period is $\lambda_{u}=2.3 \mathrm{~cm}$, which may be difficult to attain a high field undulator magnet. The wiggler number becomes very small, and the required laser amplification power becomes very large (see Fig. 6). If there is a longer wavelength high bandwidth laser, e.g. $\lambda=10 \mu \mathrm{m}$, the undulator period becomes $23 \mathrm{~cm}$, and the required laser amplification power will be greatly reduced as shown in Fig. 7 . Although it may still require $80 \mathrm{~W}$ of laser amplification power to attain a $1 \mathrm{hr}$ cooling time (for $B_{u}=6 \mathrm{~T}$ ), this is dramatically improved in comparison with the $1000 \mathrm{~W}$ requirement shown in Fig. 6.

osc-talk: submitted to World Scientific on October 23, 2002 


\section{Conclusion}

In this paper, we derived a necessary condition for the transverse phase space damping in the optical stochastic cooling. We have also explored the damping rates, the amplification factor, cooling dynamics, and the required peak and average output power of the laser. We derived an optimal laser focusing condition for the charged particle beam and the laser beam interaction in an undulator. With the available optical amplifiers at the present, it is rather impractical to use the optical stochastic cooling method to cool proton and heavy ion beams at very high energies. However, we find that the cooling method may be beneficial to low energy electron beams, and around $1 \mathrm{TeV}$ proton beams.

We also point out the difficulties of OSC with optimal gain condition. At the optimal gain, the required laser power is usually very large. As the beam is cooled, it is difficult to change the charged particle optics for a larger $k I_{D}$ to compensate the decrease in emittances. The best solution is to cool beams in the low gain regime, where the heating term may be negligible. For the TEVATRON, it seems to be feasible to use the Ti-Saphire $\lambda=0.78 \mu \mathrm{m}$ for OSC at $1 \mathrm{TeV}$. One needs a shorter wavelength broadband laser for VLHC, and a long wavelength broadband laser for the RHIC.

In actual implementation of the OSC, one should also consider the efficiency of laser pumping and optical transmission, the linearity of the laser amplification, noise, etc. These problems can be considered if there is a realistic project to carry out experimental tests.

\section{References}

1. M. Zolotorev and A. Zholents, Phys. Rev. E, 50, p.3087 (1994).

2. S. van der Meer, Stochastic damping of betatron oscillations, CERN/ISR PO/72-31 (unpublished), (1972); D. Möhl, CERN Accelerator School Report, CERN 87-03, p.453 (1987).

3. S.Y. Lee, Y.K. Zhang, and K.Y. Ng, submitted to Phys. Rev. E for publication.

4. see for example, S.Y. Lee, Accelerator Physics, (World Scientific Inc., Singapore, 1999).

5. C. Pellegrini and I.E. Campisi, AIP conference Proceedings, 105, p. 1062 (1983); E.D. Courant, C. Pellegrini, and W. Zakowicz, Phys. Rev. A 32, $2813(1985)$.

6. If we assume that the photon beam be distributed as bi-Gaussian radially but uniformly along the longitudinal $s$-direction, the total energy of the photons can be written as

$$
W_{0}=\int \frac{W_{0} / \Delta s}{2 \pi \sigma_{x} \sigma_{z}} \exp \left(-\frac{x^{2}}{2 \sigma_{x}^{2}}-\frac{z^{2}}{2 \sigma_{z}^{2}}\right) d x d z \Delta s,
$$

where the energy density is $\frac{1}{2} \epsilon_{0} \mathcal{E}_{2}^{2}=\left(W_{0} / \Delta s\right) /\left(2 \pi \sigma_{x} \sigma_{z}\right)$ and $\sigma_{x}$ and $\sigma_{z}$ are the spreads in the transverse directions. Here $\mathcal{E}_{2}$ is the peak field at $x=0$ and $z=0$. Now, we can write $W_{0}=\frac{1}{2} \epsilon_{0} \mathcal{E}_{2}^{2} A_{2} \Delta s$, i.e. the effective photon beam area is $A_{2}=2 \pi \sigma_{x} \sigma_{z}$. For a photon beam with cylindrical symmetry, we find $A_{2}=2 \pi \sigma_{r}^{2}$.

osc-talk: submitted to World Scientific on October 23, 2002 
7. The emittance of the photon beam, $\lambda /(4 \pi)$, may substantially differ from the emittance of the charged particle beams, e.g. $3.3 \mathrm{~nm}$ for the TEVATRON at $1 \mathrm{TeV}$ and $16 \mathrm{~nm}$ for the RHIC beam at $100 \mathrm{GeV} / \mathrm{amu}$. The efficiency of the cooling may be reduced by the overlap area between the charged particle and the photon beams. The optimal energy gain at the second undulator for the charged particle beams is equivalent to the minimum in the laser power.

8. At high energy, the self-consistent solution of $\lambda=\lambda_{u}\left(2+K^{2}\right) /\left(4 \gamma^{2}\right)$ and $K=$ $q B_{u} \lambda_{u} /(2 \pi m c)$ leads to a conclusion that the peak power of the laser amplifier is proportional to $\gamma^{2}$ of Eq. (30). The minimum power requirement occurs at $\gamma_{\text {min }} \sim 1.14 \sqrt{\pi m c /\left(q B_{u} \lambda\right)}$. 